\title{
A representative sample of Be stars III: $H$ band spectroscopy
}

\author{
I. A. Steele ${ }^{1}$ and J. S. Clark ${ }^{2,3}$ \\ 1 Astrophysics Research Institute, Liverpool John Moores University, Liverpool CH41 1LD, UK \\ 2 Astronomy Centre, CPES, University of Sussex, Brighton BN1 9QH, UK \\ ${ }^{3}$ Dept. of Physics \& Astronomy, University College London, London WC1E 6BT, UK
}

Received 24 January 2000 / Accepted 6 March 2001

\begin{abstract}
We present $H$ band $(1.53 \mu \mathrm{m}-1.69 \mu \mathrm{m})$ spectra of 57 isolated Be stars of spectral types O9-B9 and luminosity classes III, IV \& V. The H i Brackett ( $n-4)$ series is seen in emission from Br-11-18, and Fe II emission is also apparent for a subset of those stars with H I emission. No emission from species with a higher excitation temperature, such as He II or C III is seen, and no forbidden line emission is present. A subset of 12 stars show no evidence for emission from any species; these stars appear indistinguishable from normal B stars of a comparable spectral type. In general the line ratios constructed from the transitions in the range Br-11-18 do not fit case B recombination theory particularly well. Strong correlations between the line ratios with $\mathrm{Br}-\gamma$ and spectral type are found. These results most likely represent systematic variations in the temperature and ionization of the circumstellar disc with spectral type. Weak correlations between the line widths and projected rotational velocity of the stars are observed; however no systematic trend for increasing line width through the Brackett series is observed.
\end{abstract}

Key words. stars: emission-line, Be - infrared: stars

\section{Introduction}

Be stars are defined as hot, non-supergiant stars that show, or have shown at some stage, emission lines in their spectrum. About $20 \%$ of B stars are Be stars hence they are an important part of the hot star population. Understanding them is crucial to our obtaining a complete picture of hot-star winds. They are rapid rotators (rotating at a mean velocity of $70 \%$ of their break-up speeds, Porter 1996) and often have complex and variable emission line profiles that at sufficient resolution are double peaked. In addition they show an optical and infrared continuum excess. In spite of many attempts, no complete explanation for the Be phenomenon has yet been found.

Observations have produced an empirical description of Be star circumstellar environments which is now generally accepted: a dense "disc" exists in the equatorial plane (probably rotating in a Keplerian fashion), whilst over the polar regions there is a fast wind (velocities up to $\sim 1000-2000 \mathrm{~km} \mathrm{~s}^{-1}$ ). The disc is ionized, and it is recombination in the disc that gives the emission lines. The double peaked structure of these lines is then due to the velocity structure and to self-absorption in the disc.

Send offprint requests to: I. A. Steele, e-mail: ias@astro.livjm.ac.uk
Gehrz et al. (1974) showed that the optical and infrared excess in the systems could be explained by disc free-free emission. Recent radio and optical interferometric data has confirmed the existence of the disc (Dougherty \& Taylor 1992; Quirrenbach et al. 1994; Stee et al. 1995).

Studies of Be stars have traditionally concentrated on optical-near IR photometry and optical spectroscopy. However, the optical H I recombination lines do not function as good diagnostics of the inner regions of the circumstellar disc. Since it is likely that studying the inner regions of the disc will result in important constraints to the physical processes giving rise to the different wind regimes the need for observations of this region are pressing. NearIR spectroscopy provides one tool to probe the innermost regions of the circumstellar disc. We have therefore obtained $H$ and $K$ band spectra of a sample of some $\sim 60 \mathrm{Be}$ stars, from B0-B9 to study this region of the circumstellar envelope for the first time.

This paper is the third of a series on the optical and near IR spectral properties of a representative sample of 58 Be stars. In Steele et al. (1999; Paper I) we discuss the the basic properties of our sample, such as spectral type, luminosity class and projected rotational velocity, and determine that no significant selection effects bias our sample. In Clark \& Steele (2000; Paper II) we present 


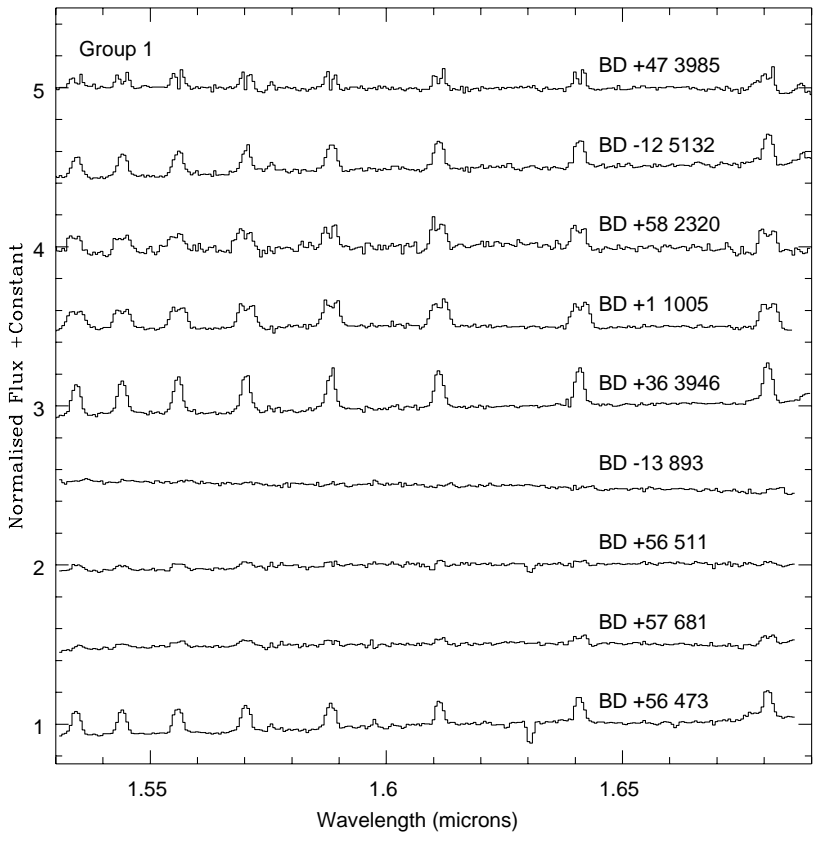

Fig. 1. $H$ band spectra for Group 1 objects (I)

the $K$ band spectra of the sample and relate these to the underlying properties of the stars. In this paper we present $H$ band $(1.53-1.69 \mu \mathrm{m})$ spectra of 54 of the stars from Paper I, plus three additional objects (see Table 7).

\section{Observations and data reduction}

The sample of target objects contains objects from $\mathrm{O} 9$ to B8.5 and of luminosity classes III (giants) to V (dwarfs), as well as three shell stars. The sample was selected in an attempt to contain several objects that were typical of each spectral and luminosity class in the above range; it therefore does not reflect the spectral and luminosity class space distribution of Be stars, but only the average properties of each subclass in temperature and luminosity. A spectral type and measure of $v \sin i$ was derived for each object in the sample and were presented in Paper I (only the spectral classifications are repeated here for sake of brevity). The distribution of $v \sin i$ within each temperature and luminosity class was carefully investigated and the conclusion drawn that there were no significant selection effects biasing the average properties of the objects (see Paper I for details).

The observations were carried out on the United Kingdom Infrared Telescope (UKIRT) on 1996 June 29 and October 1-2 (see Tables 2-7), using the Cooled Grating Spectrometer (CGS4). The observations were made using the short focal length camera plus the 150 line/mm grating, giving an ideal coverage from 1.53 to $1.71 \mu \mathrm{m}$ with a velocity resolution of $\sim 100 \mathrm{~km} \mathrm{~s}^{-1}$. Unfortunately, due to problems with the slit rotation mechanism, the wavelength coverage was slightly curtailed, leading to a final wavelength coverage of 1.53 to $1.69 \mu \mathrm{m}$, excluding the He I $1.7004 \mu \mathrm{m}$ transition. Data reduction was carried out in a similar manner as described

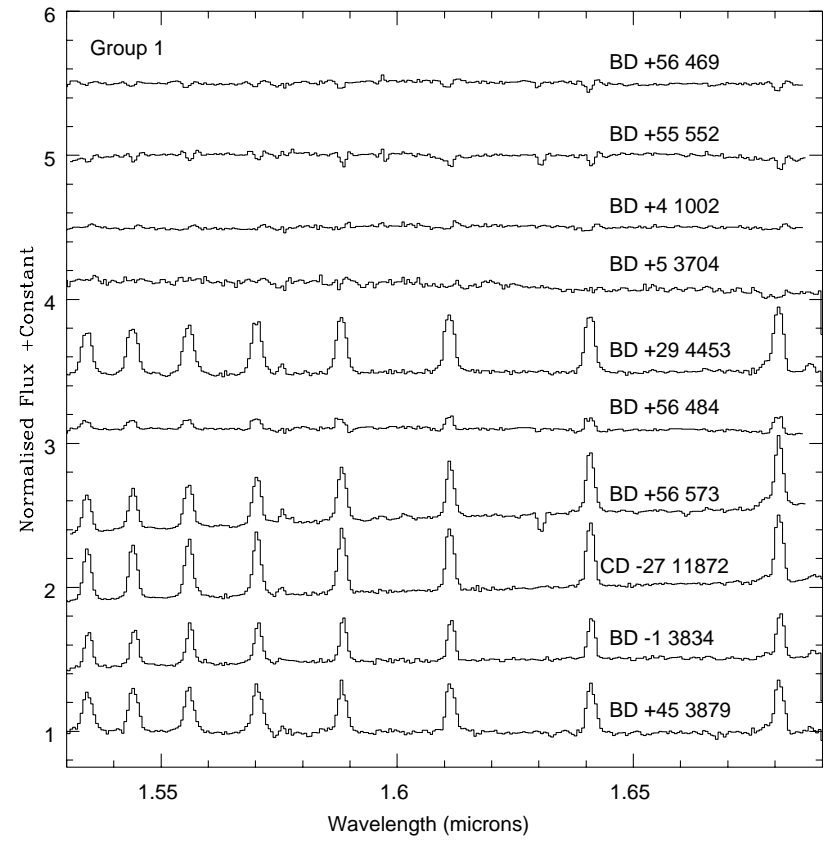

Fig. 2. $H$ band spectra for Group 1 objects (II)

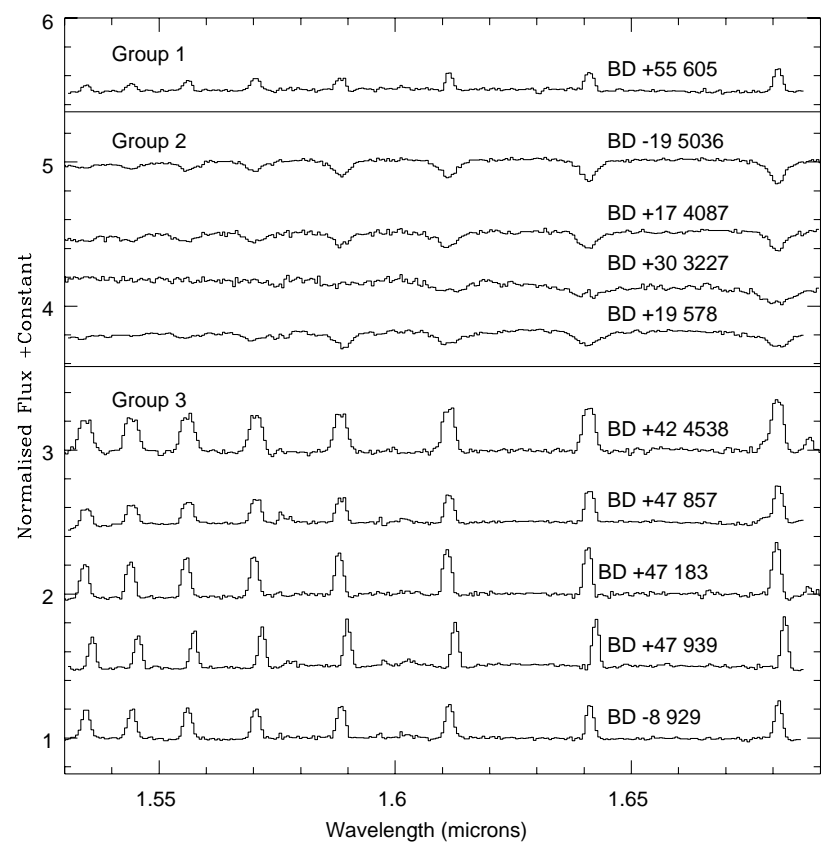

Fig. 3. $H$ band spectra for Group 1 (top panel) objects (III), Group 2 (middle panel) objects and Group 3 objects (bottom panel)

in Paper II with correction for telluric features by ratioing observed G type standards with the solar spectrum to remove features within their photosphere, and then ratioing the object spectrum with the modified standard spectrum.

\section{Discussion}

\subsection{Line identification}

The primary goal of these observations was to observe the $\mathrm{H}$ I Brackett recombination series. The wavelength 
Table 1. $K$ Band Spectral Groups and their corresponding optical spectral types (see Paper II for details)

\begin{tabular}{lll}
\hline Group & $K$ Band appearance & Spectral Type \\
\hline 1 & $\mathrm{Br} \gamma$ emission, He I features & O9e - B3e \\
2 & $\mathrm{Br} \gamma$ absorption, He I features & O9 $-\mathrm{B} 3$ \\
3 & $\mathrm{Br} \gamma+\mathrm{Mg}$ II emission, no He I features & $\mathrm{B} 2 \mathrm{e}-\mathrm{B} 4 \mathrm{e}$ \\
4 & $\mathrm{Br} \gamma$ absorption, no He I or $\mathrm{Mg}$ II features & $\mathrm{B} 4-\mathrm{B} 9$ \\
5 & $\mathrm{Br} \gamma$ emission, no He I or $\mathrm{Mg}$ II features & $\mathrm{B} 4 \mathrm{e}-\mathrm{B} 9 \mathrm{e}$ \\
\hline
\end{tabular}

Table 2. Summary of measured H I Brackett series, He I and Fe II equivalent widths in $\AA$ for Group 1 stars. Note that no correction is made in these tables for the underlying photospheric absorption line, and that a positive EW corresponds to emission. $e$ indicates emission in the relevant transition that is to weak to measure (typically $<0.2 \AA$ ), the addition of a question mark represents an uncertainty as to its presence. A dash indicates that the feature was not within the wavelength range of that particular spectrum. The estimated error in the equivalent widths is $20 \%$. The spectral type of each star as determined in Paper I is given in Col. 2 (where an italicized type indicates that only a historical classification is available), and the observation date during 1996 is indicated in Col. 3

\begin{tabular}{lllllllllllccc}
\hline Object & Spec. & Obs. & Br18 & Br17 & Br16 & Br15 & Fe II & Br14 & Br13 & Br12 & Fe II & Br11 & Fe II \\
& Type & Date & & & & & 1.576 & & & & 1.679 & \\
\hline CD -27 11872 & B0.5V-III & $29 / 6$ & 8.3 & 9.2 & 9.8 & 10.0 & 0.8 & 10.0 & 9.7 & 9.6 & $\mathrm{e}$ & 9.6 & $\mathrm{e}$ \\
BD -13 893 & B1-3V & $1 / 10$ & 0.0 & 0.0 & 0.0 & 0.0 & 0.0 & 0.0 & 0.0 & 0.2 & 0.0 & 1.4 & - \\
BD -12 5132 & BN0.2III & $29 / 6$ & 2.8 & 3.1 & 3.5 & 3.9 & 1.0 & 4.7 & 4.6 & 4.1 & $\mathrm{e}$ & 3.9 & $\mathrm{e}$ \\
BD -1 3834 & B2IV & $29 / 6$ & 4.6 & 5.3 & 5.6 & 6.2 & 0.8 & 6.3 & 6.2 & 5.7 & $\mathrm{e}$ & 5.6 & $\mathrm{e}$ \\
BD +1 1005 & B1-3V & $2 / 10$ & 4.9 & 5.0 & 5.5 & 6.3 & 0.0 & 6.1 & 5.9 & 5.6 & 0.0 & 5.7 & - \\
BD +4 1002 & B2-3III & $2 / 10$ & 0.2 & 0.2 & 0.3 & 0.2 & 0.0 & 0.3 & 0.7 & 0.1 & 0.0 & 0.1 & - \\
BD +5 3704 & B2.5V & $29 / 6$ & 0.0 & 0.0 & 0.0 & 0.1 & 0.0 & 0.2 & 0.0 & 0.0 & 0.0 & -0.2 & 0.0 \\
BD +29 4453 & B1.5V & $29 / 6$ & 8.7 & 9.7 & 9.9 & 10.2 & 0.9 & 10.6 & 11.5 & 10.5 & $\mathrm{e}$ & 11.7 & $\mathrm{e}$ \\
BD +36 3946 & B1V & $29 / 6$ & 4.6 & 5.5 & 5.5 & 5.6 & 0.4 & 6.0 & 5.7 & 5.5 & $\mathrm{e}$ & 5.4 & $\mathrm{e}$ \\
BD +45 3879 & B1.5V & $29 / 6$ & 7.8 & 7.8 & 7.8 & 8.5 & 0.7 & 7.7 & 8.4 & 7.8 & $\mathrm{e}$ & 9.3 & $\mathrm{e} ?$ \\
BD +47 3985 & B1-2sh & $29 / 6$ & 1.8 & 2.1 & 2.4 & 1.9 & 0.2 & 1.8 & 2.1 & 2.4 & $\mathrm{e}$ & 4.1 & $\mathrm{e}$ \\
BD +55 605 & B1V & $1 / 10$ & 0.7 & 0.9 & 1.4 & 2.1 & 0.0 & 2.1 & 2.4 & 2.9 & 0.0 & 3.2 & - \\
BD +55 552 & B4V & $1 / 10$ & 0.1 & -0.4 & -0.1 & -1.2 & 0.0 & -1.0 & -1.1 & -3.0 & 0.0 & -0.3 & - \\
BD +56 469 & B0-2III & $1 / 10$ & -0.4 & -0.4 & -0.3 & -0.3 & 0.0 & -0.3 & -0.3 & -0.6 & 0.0 & -0.6 & - \\
BD +56 473 & B1V-III & $2 / 10$ & 3.4 & 3.5 & 3.7 & 3.9 & 0.5 & 4.0 & 3.9 & 3.8 & $\mathrm{e}$ & 4.5 & - \\
BD +56 478 & B1.5V & $1 / 10$ & 0.9 & 1.2 & 1.4 & 1.5 & 0.0 & 1.5 & 1.4 & 1.9 & 0.0 & 2.1 & - \\
BD +56 511 & B1III & $1 / 10$ & 0.5 & 0.4 & 0.7 & 1.0 & 0.0 & 0.1 & 0.3 & 0.7 & 0.0 & 0.3 & - \\
BD +56 573 & B1.5V & $1 / 10$ & 5.9 & 7.2 & 7.8 & 8.6 & 2.1 & 9.8 & 8.8 & 9.7 & $\mathrm{e}$ & 10.3 & - \\
BD +57 681 & B0.5V & $1 / 10$ & 0.6 & 0.4 & 0.9 & 1.2 & 0.0 & 0.9 & 1.0 & 1.6 & 0.0 & 2.0 & - \\
BD +58 2320 & B2V & $29 / 6$ & 2.9 & 3.6 & 4.1 & 4.0 & 0.0 & 3.2 & 4.6 & 3.8 & 0.0 & 5.9 & 0.0 \\
\hline
\end{tabular}

Table 3. Summary of H I Brackett series for Group 2 stars. See Table 2 for explanation

\begin{tabular}{lllccccccccccc}
\hline Object & Spec. & Obs. & Br18 & Br17 & Br16 & Br15 & Fe II & Br14 & Br13 & Br12 & Fe II & Br11 & Fe II \\
& Type & Date & & & & & 1.576 & & & & 1.679 & \\
\hline CD -25 12642 & B0.7III & $29 / 6$ & -0.3 & -0.5 & -0.4 & -0.8 & 0.0 & -1.7 & -2.9 & -3.0 & 0.0 & -4.0 & 0.0 \\
BD +20 4449 & B0III & $29 / 6$ & 0.0 & 0.0 & 0.0 & -0.5 & 0.0 & -1.0 & -1.7 & -2.0 & 0.0 & -3.0 & 0.0 \\
BD +25 4083 & B0.7III & $29 / 6$ & -0.4 & -0.2 & -0.1 & -0.7 & 0.0 & -1.5 & -2.1 & -2.5 & 0.0 & -3.2 & 0.0 \\
BD +28 3598 & O9II & $29 / 6$ & 0.0 & 0.0 & 0.0 & 0.0 & 0.0 & -0.3 & 0.3 & -0.7 & 0.0 & -1.5 & 0.0 \\
BD +29 3842 & B1II & $29 / 6$ & -0.3 & -0.3 & -0.3 & -0.8 & 0.0 & -1.4 & -1.6 & -2.3 & 0.0 & -2.3 & 0.0 \\
BD +37 3856 & B0.5V & $29 / 6$ & 0.0 & 0.0 & 0.0 & -0.5 & 0.0 & -0.8 & -1.7 & -2.3 & 0.0 & -2.8 & 0.0 \\
BD +45 933 & B1.5V & $1 / 10$ & -0.5 & -0.9 & -1.1 & -1.6 & 0.0 & -1.8 & -1.3 & -2.0 & 0.0 & -1.9 & - \\
BD +56 493 & B1V & $1 / 10$ & -0.3 & -0.1 & -0.2 & -0.7 & 0.0 & -1.3 & -2.3 & -1.7 & 0.0 & -2.9 & - \\
\hline
\end{tabular}

Table 4. Summary of H I Brackett series for Group 3 stars. See Table 2 for explanation

\begin{tabular}{|c|c|c|c|c|c|c|c|c|c|c|c|c|c|}
\hline Object & $\begin{array}{l}\text { Spec. } \\
\text { Type }\end{array}$ & $\begin{array}{l}\text { Obs. } \\
\text { Date }\end{array}$ & Br18 & Br17 & Br16 & Br15 & $\begin{array}{c}\text { Fe II } \\
1.576\end{array}$ & Br14 & Br13 & Br12 & $\begin{array}{c}\text { Fe II } \\
1.679\end{array}$ & Br11 & $\begin{array}{c}\text { Fe II } \\
1.687\end{array}$ \\
\hline BD -8 929 & $B 2 V$ & $2 / 10$ & 4.6 & 4.5 & 4.5 & 4.6 & 0.0 & 5.0 & $\overline{5.2}$ & 4.6 & 0.0 & 4.9 & - \\
\hline $\mathrm{BD}+424538$ & B2.5 & $29 / 6$ & 8.2 & 8.7 & 9.2 & 8.4 & 0.0 & 9.0 & 9.3 & 9.2 & 0.0 & 10.6 & 0.0 \\
\hline $\mathrm{BD}+47183$ & $\mathrm{~B} 2.5 \mathrm{~V}$ & $29 / 6$ & 5.6 & 5.6 & 5.7 & 5.8 & 0.3 & 5.6 & 6.4 & 6.3 & $\mathrm{e}$ & 7.0 & $\mathrm{e}$ \\
\hline $\mathrm{BD}+47857$ & B4IV & $1 / 10$ & 2.4 & 3.5 & 4.3 & 4.4 & 1.5 & 4.4 & 4.5 & 5.3 & $\mathrm{e}$ & 5.7 & - \\
\hline $\mathrm{BD}+47939$ & $\mathrm{~B} 2.5 \mathrm{~V}$ & $1 / 10$ & 3.6 & 4.1 & 4.3 & 4.6 & 0.9 & 5.4 & 5.2 & 5.2 & e & 6.4 & - \\
\hline
\end{tabular}


Table 5. Summary of H I Brackett series for Group 4 stars. See Table 2 for explanation

\begin{tabular}{llllllllllllcc}
\hline Object & Spec. & Obs. & Br18 & Br17 & Br16 & Br15 & Fe II & Br14 & Br13 & Br12 & Fe II & Br11 & Fe II \\
& Type & Date & & & & & 1.576 & & & & 1.679 & 1.687 \\
\hline BD -19 5036 & B4III & $29 / 6$ & -0.8 & -1.4 & -2.0 & -2.5 & 0.0 & -4.8 & -5.4 & -6.3 & 0.0 & -7.5 & 0.0 \\
BD +17 4087 & B6III-V & $29 / 6$ & -1.2 & -1.4 & -2.1 & -2.9 & 0.0 & -3.5 & -5.1 & -5.2 & 0.0 & -5.7 & 0.0 \\
BD +19 578 & B8V & $1 / 10$ & -0.3 & -0.4 & -0.4 & -1.2 & 0.0 & -2.1 & -4.1 & -5.3 & 0.0 & -5.9 & - \\
BD +30 3227 & B4V & $29 / 6$ & 0.0 & 0.0 & 0.0 & -1.0 & 0.0 & -3.4 & -5 & -5.7 & 0.0 & -7.5 & 0.0 \\
\hline
\end{tabular}

Table 6. Summary of H I Brackett series for Group 5 stars. See Table 2 for explanation

\begin{tabular}{lllllllllllccc}
\hline Object & Spec. & Obs. & Br18 & Br17 & Br16 & Br15 & Fe II & Br14 & Br13 & Br12 & Fe II & Br11 & Fe II \\
& Type & Date & & & & & 1.576 & & & & 1.679 & 1.687 \\
\hline CD -27 13183 & B7V & $29 / 6$ & 0.0 & 0.0 & -1.0 & -1.4 & 0.0 & -2.0 & -3.1 & -3.2 & 0.0 & -4.80 & 0.0 \\
BD -20 5381 & B5V & $29 / 6$ & 1.0 & 1.0 & 1.7 & 1.1 & 0.0 & 0.7 & 1.4 & 0.7 & 0.0 & 0.8 & - \\
BD -0 3543 & B7V & $29 / 6$ & 0.0 & 0.0 & 0.0 & -1.2 & 0.0 & -2.2 & -2.4 & -2.7 & 0.0 & -3.3 & 0.0 \\
BD +0 1203 & B5III & $29 / 6$ & 1.1 & 0.7 & 0.7 & 0.8 & 0.0 & 0.9 & 0.8 & 1.2 & 0.0 & 2.0 & 0.0 \\
BD +2 3815 & B7-8sh & $29 / 6$ & -0.5 & -0.8 & -1.2 & -2.0 & 0.0 & -3.7 & -3.5 & -4.1 & 0.0 & -4.6 & 0.0 \\
BD +21 4695 & B6III-V & $29 / 6$ & 0.0 & -0.3 & -0.8 & -1.3 & 0.0 & -2.8 & -3.4 & -5.4 & 0.0 & -6.4 & 0.0 \\
BD +27 3411 & B8V & $29 / 6$ & 0.0 & -0.4 & -0.7 & -1.1 & 0.0 & -2.4 & -4.5 & -5.6 & 0.0 & -6.5 & 0.0 \\
BD +37 675 & B7V & $1 / 10$ & 1.2 & 0.6 & 1.0 & 0.0 & e & -0.2 & -1.7 & -2.0 & e & -1.5 & - \\
BD +43 1048 & B6IIIsh & $1 / 10$ & 0.2 & 0.2 & 0.2 & 0.3 & 0.0 & 0.5 & 0.7 & 1.4 & 0.0 & 1.2 & - \\
BD +46 275 & B5III & $2 / 10$ & -0.1 & -0.9 & -2.2 & -2.4 & 0.0 & -4.9 & -4.3 & -5.2 & 0.0 & -6.0 & - \\
BD +49 614 & B5III & $1 / 10$ & 0.2 & 0.3 & 0.6 & 0.5 & 0.0 & -1.6 & -3.3 & -2.7 & 0.0 & -2.7 & - \\
BD +50 825 & B7V & $2 / 10$ & 0.3 & 0.3 & 0.2 & -1.0 & 0.0 & -0.2 & -1.7 & -2.0 & 0.0 & -1.5 & - \\
BD +50 3430 & B8V & $29 / 6$ & 0.5 & 0.5 & 0.6 & -1.7 & 0.0 & -1.7 & -1.9 & -2.6 & 0.0 & -4.5 & 0.0 \\
BD +51 3091 & B7III & $29 / 6$ & -1.0 & -1.8 & -2.4 & -3.8 & 0.0 & -4.3 & -6.2 & -6.8 & 0.0 & -6.7 & 0.0 \\
BD +53 2599 & B8V & $29 / 6$ & -1.1 & -1.9 & -1.9 & -2.7 & 0.0 & -2.4 & -3.7 & -5.1 & 0.0 & -7.5 & 0.0 \\
BD +55 2411 & B8.5V & $29 / 6$ & -0.4 & -0.6 & -1.2 & -2.1 & 0.0 & -4.0 & -5.0 & -6.0 & 0.0 & -7.5 & 0.0 \\
BD +58 554 & B7V & $1 / 10$ & 0.0 & 0.8 & 1.2 & 0.7 & 0.0 & 0.2 & 0.7 & 0.6 & 0.0 & 0.3 & - \\
\hline
\end{tabular}

Table 7. Summary of H I Brackett series for new stars. See Table 2 for explanation

\begin{tabular}{lllllllccccccc}
\hline Object & Spec. & Obs. & Br18 & Br17 & Br16 & Br15 & Fe II & Br14 & Br13 & Br12 & Fe II & Br11 & Fe II \\
& Type & Date & & & & & 1.576 & & & & 1.679 & 1.687 \\
\hline BD +54 2348 & B2V & $29 / 6$ & 6.2 & 7.0 & 7.3 & 7.9 & 0 & 7.6 & 7.7 & 7.9 & 0.0 & 8.7 & 0.0 \\
BD +54 2718 & B2 III & $29 / 6$ & -0.5 & -0.4 & 0.0 & 0.0 & -0.7 & -1.0 & -0.9 & -1.4 & 0.0 & -3.0 & 0.0 \\
MWC 659 & BOIIIpe & $29 / 6$ & 7.8 & 8.9 & 9.8 & 9.4 & 0.8 & 10.7 & 10.3 & 10.9 & $\mathrm{e}$ & 11.3 & $\mathrm{e}$ \\
\hline
\end{tabular}

range chosen encompasses $\mathrm{Br}-18-11$, as well as Fe II lines at $1.534,1.600$ and $1.620 \mu \mathrm{m}$ (amongst others). Various He II transitions are also found within this wavelength region, however given their absence in the $K$ band spectra presented in Paper II, we do not expect to see these in emission. The spectra are presented in Figs. 1-6, and are presented in the same Groups we defined in Paper II, which were based on their $K$ band spectral morphology (see Table 1). This approach has been adopted, rather than grouping the spectra on the basis of their optically derived spectral classifications since one aim of this work was to define a classification scheme based on near-IR spectroscopy alone, for use in the classification of heavily obscured stars, such as those often found in High Mass $\mathrm{X}$-ray binary systems.

Unsurprisingly, based on the features present in the $K$ band spectra, the obvious lines seen in emission are Br18-11, and Fe II $1.576 \mu \mathrm{m}$ and $1.687 \mu \mathrm{m}$. A further feature in the blue wing of $\operatorname{Br}-11(\lambda \sim 1.678 \mu \mathrm{m})$ is also seen in a subset of spectra, the identity of which is discussed further below. No emission from species of higher excitation (such as He II, N III or C III) was observed. The presence and equivalent widths $(E W)$ of these features are summarised in Tables 2-7. Equivalent widths and FWHM were measured using the ABLINE routine of the FIGARO software package. This measures $E W$ by integrating the 


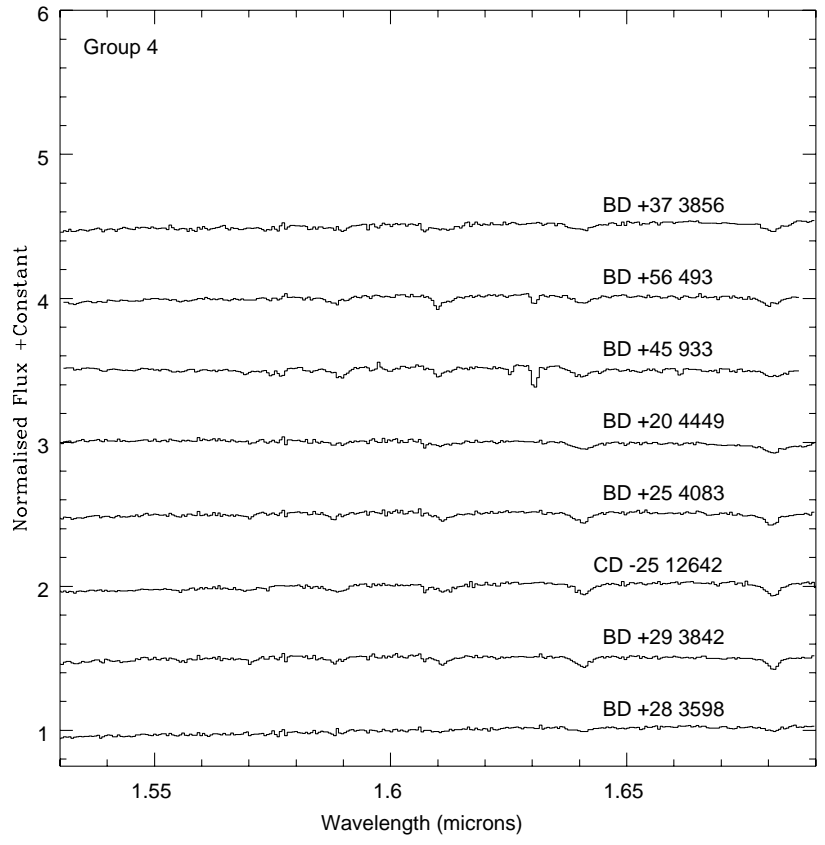

Fig. 4. $H$ band spectra for Group 4 objects

line flux relative to an interpolated continuum produced by polynomial fitting in the vicinity of the line - it therefore makes no assumption about line shape. FWHM are measured by looking at the line width which encompasses $68 \%$ of the line flux and multiplying by 1.18 . For a line of Gaussian profile this gives exactly the $F W H M$, and for any reasonably centrally concentrated profile (as ours are) it gives a result very close to the $F W H M$ that is more robust than simple Gaussian fitting.

Brackett series emission is seen in all stars of Groups 1, 3 and 5 (which all possess $\operatorname{Br} \gamma$ emission), with the possible exception of BD +51 3091, a B7 III star (Fig. 6). Of Groups 2 and 4, which were defined on the basis of the absence of $\operatorname{Br} \gamma$ emission in the $K$ band spectra, only BD +30 3227 appears to show evidence for partial infilling of Br-11-14 (Fig. 3), while the remainder appear to possess pure absorption spectra.

Fe II $1.576 \mu \mathrm{m}$ emission is seen in a total of 13 stars, compared to a total of 19 stars showing Fe II $2.089 \mu \mathrm{m}$ emission. Of the 33 stars where the wavelength coverage encompassed Fe II $1.688 \mu \mathrm{m}$, the line was in emission in 6 stars, 5 of which are Group 1 objects, the final star, MWC 659 , being one of the 3 objects without corresponding $K$ band observations (see Tables $2-7$ ). We note that all stars with Fe II $1.576 \mu \mathrm{m}$ emission also show Fe II $1.688 \mu \mathrm{m}$ emission. The correlation between the presence of Fe II emission in the $H$ and $K$ bands is weaker, with a total of 8 stars showing Fe II emission in either the $H$ or $K$ band, but not in the other. However, as with the $K$ band spectra, Fe II emission only occurs in the $H$ band spectra of those stars with strong Brackett series emission; $E W_{\mathrm{Br}-11}>3.5 \AA^{1}\left(E W_{\mathrm{Br} \gamma}>8 \AA\right)$.

\footnotetext{
1 Note that in this paper we will employ the convention that positive equivalent widths indicate emission features.
}

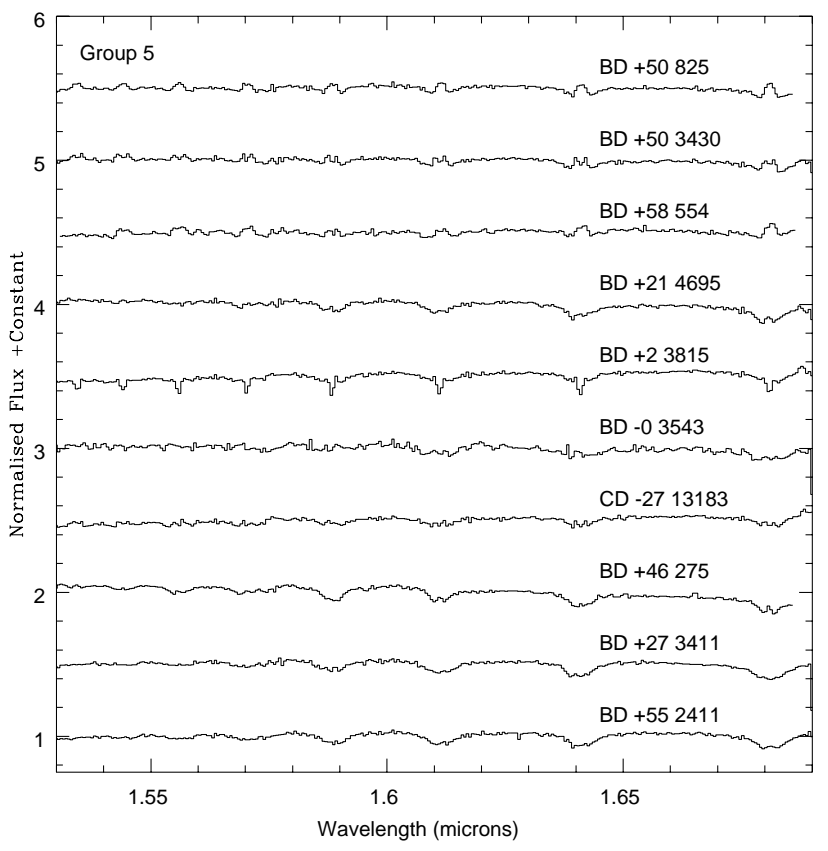

Fig. 5. $H$ band spectra for Group 5 objects (I)

Two possible identifications exist for the emission feature at $\sim 1.678 \mu \mathrm{m}$; Fe II $1.679 \mu \mathrm{m}$ and [Fe II] $1.678 \mu \mathrm{m}$. Of these, we favour Fe II $1.679 \mu \mathrm{m}$ since the feature is only seen in stars that show emission in the other two Fe II lines, and no emission is seen in the (albeit weaker) [Fe II] transitions at $1.534 \mu \mathrm{m}$ and $1.644 \mu \mathrm{m}$ (Hamann \& Persson 1989).

Overall, as regards spectral classification using the $H$ band spectra of Be stars, it is apparent that with the absence of many pure photospheric features that are uncontaminated by disc emission it is only possible to perform a very general spectral classification into "early" (B0eB4e) and "late" (B5e-B9e) spectral types. The problem of the lack of photospheric features uncontaminated by emission from the circumstellar disc is compounded by the (expected) lack of emission from species with a wide range of excitation energies (although we speculate that the presence of He I $1.700 \mu \mathrm{m}$ emission may function as an additional, valuable diagnostic of early spectral types).

\subsection{Brackett line strengths}

In order to make an accurate comparison of Brackett line strengths between objects of different spectral type, it is necessary to remove the effect of the underlying photospheric absorption lines from the spectra. To carry out this correction we used the equivalent widths for normal (non-emission) B stars presented for Br- $\gamma$ by Hanson et al. (1996) and for Br 11-18 by Steele \& Clark (2001). The correction itself simply consisted of subtracting the equivalent width derived from a least squares fit to the appropriate transition for the normal B stars from the emission line equivalent width. The discussion and figures in this section therefore refer to this corrected equivalent width. We note 


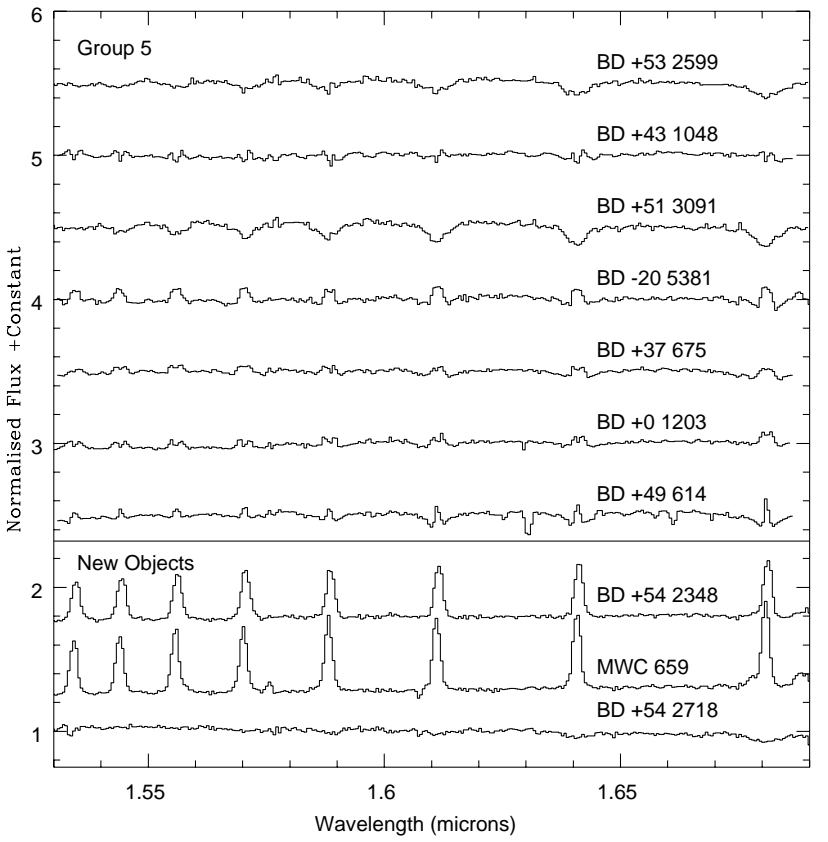

Fig. 6. $H$ band spectra for Group 5 (upper panel) objects (II) and new objects (lower panel)

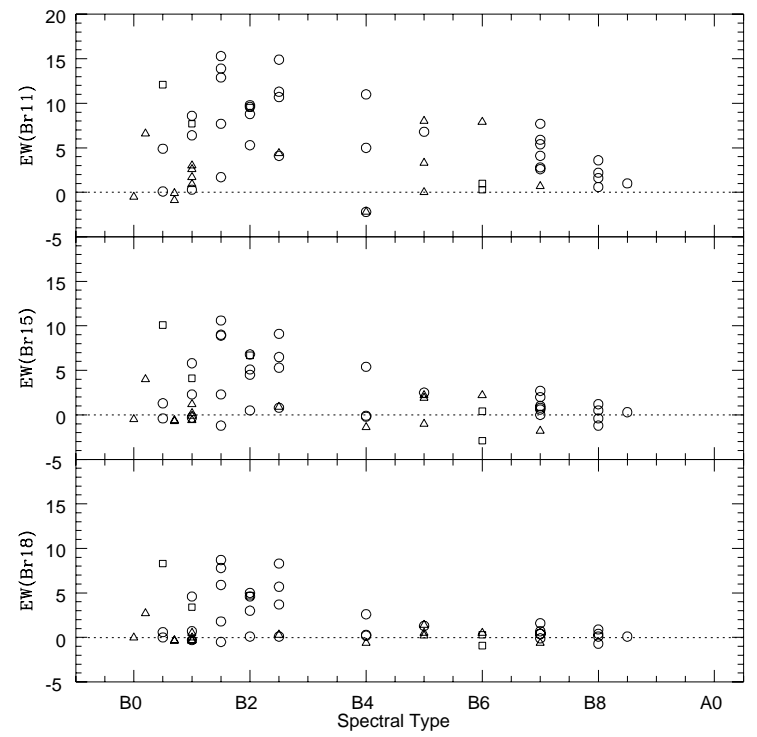

Fig. 7. Equivalent width $(E W)$ of Br-11 (top panel), Br-15 (middle panel ) and $\mathrm{Br}-18$ (bottom panel) in $\AA$ (where positive $E W$ 's indicate emission) against spectral type. The equivalent widths have been corrected for the underlying photospheric absorption as indicated in the text. Triangular symbols represent luminosity class III, squares luminosity class IV and circles luminosity class $\mathrm{V}$

that within the small wavelength range encompassed by Br-11 to Br-18, these corrected equivalent widths may be divided by one another and treated as flux ratios without introducing significant error.

We plot the $E W$ of $\mathrm{Br}-11,15 \& 18$ against spectral type in Fig. 7 (the remainder of the lines are not plotted due to reasons of space). As with $\operatorname{Br} \gamma$ (Paper II) we find that while a linear correlation between spectral

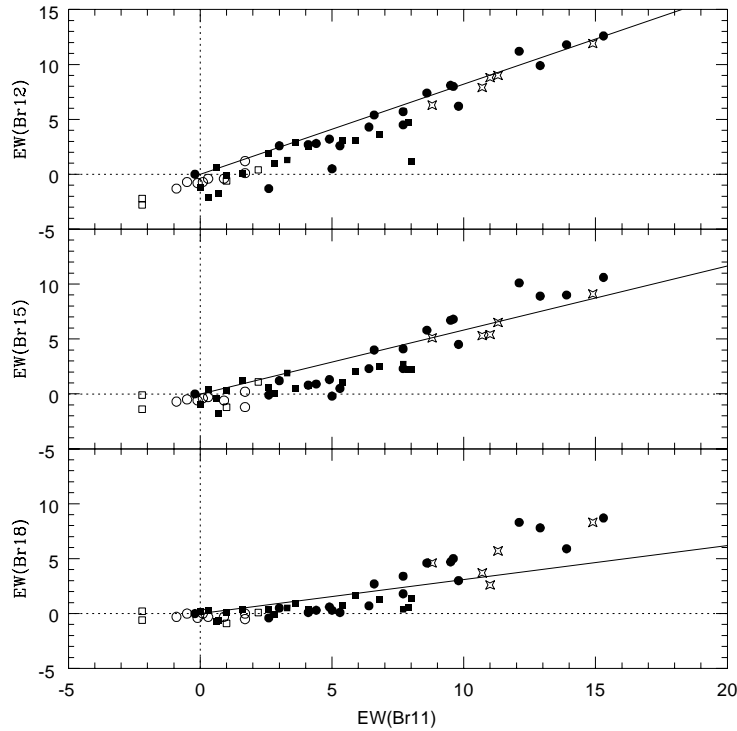

Fig. 8. $E W$ of Br-11 against Br-12 (top panel), Br-15 (middle panel) and Br-18 (bottom panel). Filled circles represent objects from Groups 1, circles Group 2, stars Group 3, empty squares Group 4 and filled squares Group 5 (see Table 1 for group definitions). The line indicates the expected line ratio for case $\mathrm{B}$ recombination

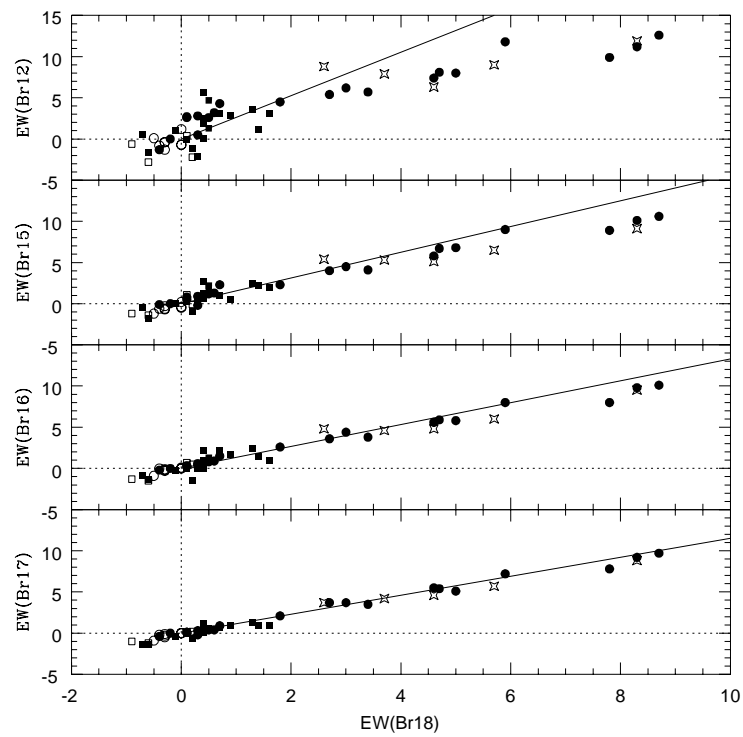

Fig. 9. $E W$ of $\mathrm{Br}-18$ against Br-12, $-15,-16$ and -17 . Symbols and line as Fig. 8

type and $E W$ is absent, a strong trend in the upper envelope of the line strengths is present, with lower mean and maximum line strengths for the later ( B $5-\mathrm{B} 9)$ spectral types. We find no evidence of systematic differences between the emission characteristics of stars of differing luminosity classes.

In Fig. 8 we plot the $E W$ of $\mathrm{Br}-11$ against those of Br-12, -15 and -18 , while in Fig. 9 we plot the $E W$ of $\mathrm{Br}-18$ versus $\mathrm{Br}-12,-15,-16$ and -17 . Note that the empty symbols cluster around $E W$ of zero in both figures, with a typical deviation of $\sim 1 \AA$. As these are non-emission line objects, this indicates that the accuracy of our 


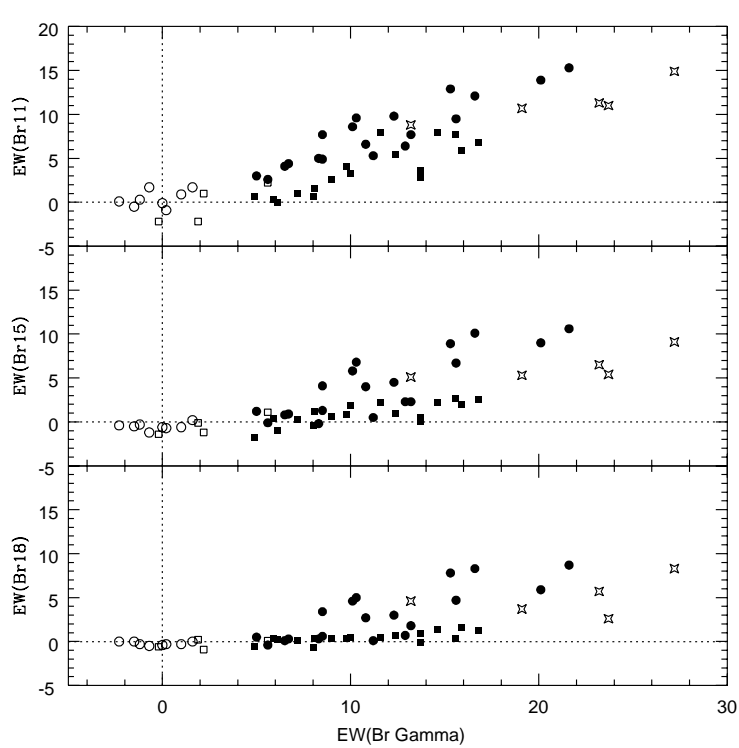

Fig. 10. Plot of the $E W$ of $\operatorname{Br} \gamma$ (data from Paper II) against Br-11 (top panel), Br-15 (middle panel) and Br-18 (bottom panel; all $E W$ in $-\AA$ ). Symbols as Fig. 8

correction for the photospheric absorption component is of that order. We also note that there appears to be no significant difference in these graphs between the various spectral groups.

Given that the $\mathrm{Br}$ 11-18 lines arise from high levels in the hydrogen atom they might be expected to be optically thin, and therefore well represented by case B recombination theory. In order to test this, we plot as a straight line in Figs. 8 and 9 the expected case B line ratios from Storey \& Hummer (1995) for ionized hydrogen. The predicted line ratios are constant to within a few per-cent for temperatures in the range $7500-40000 \mathrm{~K}$ and electron densities in the range $10^{10}-10^{14} \mathrm{~cm}^{-3}$, and are therefore should not be sensitive to these conditions within the disk. From the figures it is apparent that although there is reasonable agreement between case B theory and observation in the line ratios between closely adjacent lines (e.g. Br-11 and $\mathrm{Br}-12$, or $\mathrm{Br}-17$ and $\mathrm{Br}-18$ ), for more separated transitions the case $\mathrm{B}$ ratios do not seem to fit the data well. This may reflect either a complex disk temperature and density profile (as discussed in the following paragraph) or that the optically thin and/or LTE assumptions for these lines are not valid. Spectra extending further down the series (e.g. to $\mathrm{Br}-22$ ) where the lines are weaker would help to resolve this question.

In Fig. 10 we plot the $E W$ of $\operatorname{Br} \gamma$ (taken from Paper II) against that of $\mathrm{Br}-11,15 \& 18$. Unlike the higher transition ratios of Figs. 2 and 3 here there are large differences in the flux ratios between the groups, with a progression in the ratio $\mathrm{Br}-15 / \mathrm{Br} \gamma$ from $\sim 0.5$ (Group 1), through $\sim 0.2$ (group 3) to $\sim 0.0$ (Group 5). This effect is a real reflection of differences in line flux and not just due to continuum differences over the larger wavelength range for the $\mathrm{Br}$ $15 / \mathrm{Br} \gamma$ equivalent width ratio. At first sight it may seem that the stronger circumstellar free-free excesses of the

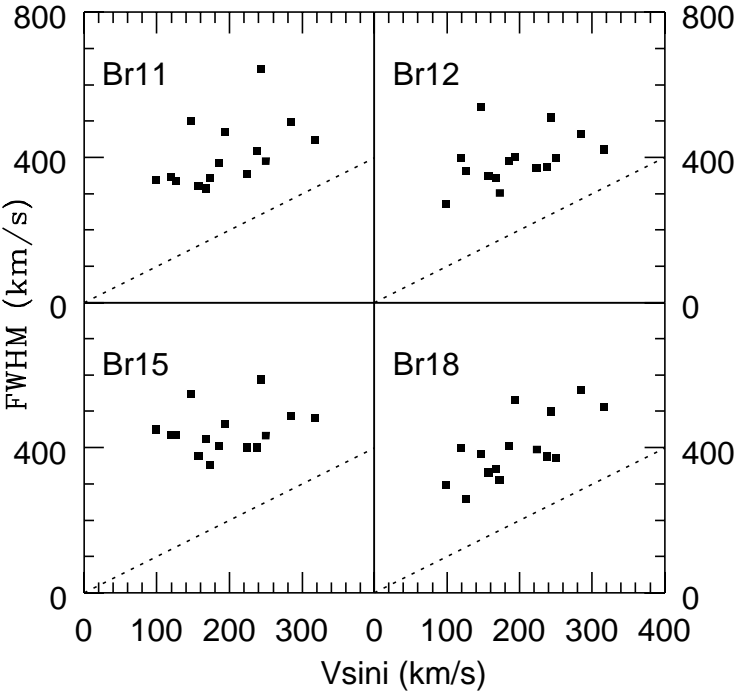

Fig. 11. Plot of the $F W H M$ (in $\AA$ ) of $\mathrm{Br}-11,12,15$ and 18 plotted against projected rotational velocity. Dotted line indicates $F W H M=v \sin i$ from Paper I

Group 1 objects could account for this effect, by reducing the $\operatorname{Br} \gamma E W$ for these objects. However this effect is small. The two continuum regions are well approximated by the photometric $H$ and $K$ bands, and Howells et al. (2001) show that the mean $E(H-K)_{\text {cs }}$ only varies from $\sim 0.12$ for Group 1 objects to $\sim 0.03$ for Group 5 objects, insufficient to cause the observed $E W$ ratio changes. We also note that this effect is somewhat mitigated by the change in intrinsic colours between Groups $1\left((H-K)_{0} \sim-0.04\right)$ and $5\left((H-K)_{0} \sim-0.01\right)$ (Koornneef 1983). We therefore believe that this progression is likely to be due to systematic changes in the temperature, degree of ionization, and structure within the discs as the temperature and flux of the underlying stars changes. Marlborough et al. (1997) present simulations of selected near-IR H I transitions for both isothermal discs, and discs with a simple radial temperature gradient, and find that varying the disc temperature does indeed lead to changes in the line decrement. However, recent work by Millar \& Marlborough (1998) shows that these simple models for the disc temperature are incorrect, and that the equilibrium temperatures for Be star discs are a complex function of disc radius, particularly in the inner disc regions responsible for nearIR recombination line emission. Further evidence for this is found in our inability to fit case B line ratios to the higher transitions as noted previously. Consequently, we defer detailed discussion of the systematic variations in the Brackett line decrement for a future paper, where modeling of the line fluxes will be accomplished for the full optical-IR dataset.

\subsection{Line widths and projected rotational velocities}

In Paper II we found correlations between the full width half maximum (FWHM) of $\mathrm{Br} \gamma$ and He I $2.058 \mu \mathrm{m}$ lines and the projected rotational velocity of the stars, in a 
similar fashion that those found for $\mathrm{H} \alpha, \beta$, and selected optical Fe II lines by Hanuschik (1989). He further found that the intrinsically weaker lines such as the higher Balmer lines and Fe II were systematically broader than $\mathrm{H} \alpha$ which he attributed to the weaker lines being produced in a smaller region of a quasi- Keplerian disc than the intrinsically stronger $\mathrm{H} \alpha$ line, where the rotational velocity would be greater, and thus the line broader. Simulations by Marlborough et al. (1997) also demonstrate the same effect, as do the longer wavelength infrared spectra of $\gamma$-Cas presented by Hony et al. (2000).

In Fig. 11 we plot the full width half maximum $(F W H M)$ of Br-11, 12, 15 and 18 for stars of Groups 1 and 3 against their projected rotational velocities from Paper 1 (stars from Group 5 were excluded due to the bias introduced into the $F W H M$ by the underlying photospheric feature). Weak correlations were found between the FWHM and the projected rotational velocities by applying Spearman's Rank Correlation to the datasets, and the results of least square fits to the data are presented in Table 7 . It is interesting to note that the minimum measured $F W H M$ is around $300 \mathrm{~km} \mathrm{~s}^{-1}$ (considerably larger than the instrumental resolution of $\left.\sim 100 \mathrm{~km} \mathrm{~s}^{-1}\right)$. This means that for the relatively slow $\left(v \sin i \sim 100-200 \mathrm{kms}^{-1}\right)$ rotators, the $F W H M$ for these lines is often more than twice the $v \sin i$ of the underlying star, possibly indicating more rapid rotation at the inner edge of the disk than at the surface of the star. We also note three Group 1 (BD +47 3985, BD +58 2320 and $\mathrm{BD}+1$ 1005) and one Group 3 (BD +42 4538) appear to have very large Br-11 $F W H M$ of greater than around $500 \mathrm{~km} \mathrm{~s}^{-1}$. However these objects also show double peaks in their line profiles, making the derivation (and interpretation) of $F W H M$ uncertain.

In Fig. 12 we plot the $F W H M$ of $\mathrm{Br}-18,15 \& 12$ vs. $\mathrm{Br}-11$. It can be seen that there is no trend to broader line profiles with higher Brackett series transitions as was suggested by the results of Hony et al. (2000). We attribute this result primarily to the narrow wavelength (and hence transition) range of our spectra compared to the work of Hony et al. (2000) whose spectra extend to the limit of each series.

\section{Conclusions}

We have analysed the $H$ band $(1.53 \mu \mathrm{m}-1.69 \mu \mathrm{m})$ spectra of 61 Be stars and found emission from H I Br11-18 and Fe II $1.576 \& 1.698 \mu \mathrm{m}$. A further emission feature at $1.679 \mu \mathrm{m}$ is present in a subset of 14 stars; this is also likely to be Fe II emission. The $\mathrm{Br} 11-18$ line ratios of non adjacent lines are not well fit by the case B assumption, and strong systematic trends in both line strength and the ratio of the higher Brackett series strengths to Br- $\gamma$ with spectral type (but not luminosity class) are observed. This is likely to be due to systematic changes in the temperature and degree of ionization between the discs of stars of early (B0-B4) and late (B5-B9) spectral type. We find that analysis of $H$ band spectra alone only
Table 8. Summary of best fits between the FWHM of Br1118 and the $v \sin i$ of the individual star. The best fit from $\operatorname{Br} \gamma$, taken from Paper II is also shown for comparison

\begin{tabular}{cc}
\hline Transition & Best Fit \\
\hline Br18 & $1.132 v \sin i+181 \mathrm{~km} \mathrm{~s}^{-1}$ \\
Br17 & $1.145 v \sin i+203 \mathrm{~km} \mathrm{~s}^{-1}$ \\
Br16 & $0.868 v \sin i+280 \mathrm{~km} \mathrm{~s}^{-1}$ \\
Br15 & $0.337 v \sin i+377 \mathrm{~km} \mathrm{~s}^{-1}$ \\
$\operatorname{Br} 14$ & $0.607 v \sin i+304 \mathrm{~km} \mathrm{~s}^{-1}$ \\
$\operatorname{Br} 13$ & $0.541 v \sin i+301 \mathrm{~km} \mathrm{~s}^{-1}$ \\
$\operatorname{Br} 12$ & $0.553 v \sin i+283 \mathrm{~km} \mathrm{~s}^{-1}$ \\
$\operatorname{Br} 11$ & $0.803 v \sin i+245 \mathrm{~km} \mathrm{~s}^{-1}$ \\
\hline $\operatorname{Br} \gamma$ & $0.759 v \sin i+149 \mathrm{~km} \mathrm{~s}^{-1}$ \\
\hline
\end{tabular}

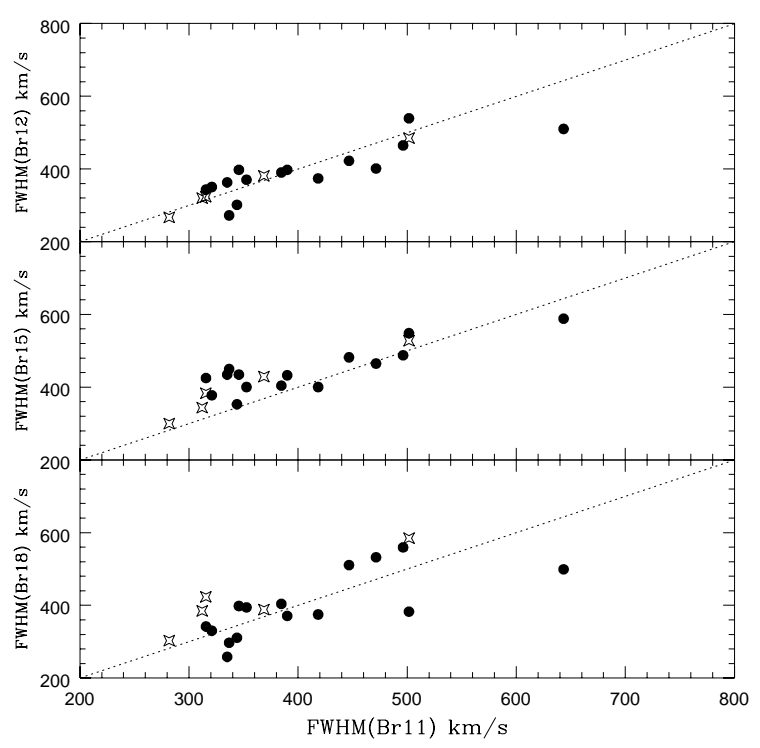

Fig. 12. $F W H M$ of $\mathrm{Br}-18,15$ \& 12 vs. Br-11 for the objects showing emission features. No systematic difference between the $F W H M$ of $\mathrm{Br}-11$, and those of the higher transitions is apparent. The larger scatter of points in the lower panel is likely to be due to the weakness of the $\mathrm{Br}-18$ line, and the consequent difficulties in obtaining an accurate measurement of the FWHM. Symbols as Fig. 8

allow the classification of stars into "early" (B0e-B4e) or late (B5e-B9e) types; no determination of the luminosity class of the object can be made. This is due to the lack of any uncontaminated photospheric features in this region, the lack of emission features encompassing a wide range of excitation temperatures and the similarity of Brackett line strengths and decrements for the early type stars. As with $\operatorname{Br} \gamma$, we find weak correlations between the $F W H M$ of Br11-18 and the projected rotational velocity of the underlying stars. We find no systematic trend in FWHM through the Brackett series down to Br-18.

Acknowledgements. UKIRT is operated by the Joint Astronomy Centre, Hawaii for the UK PPARC. We thank the support astronomers and staff of UKIRT for their invaluable 
assistance at the telescope. Data reduction and analysis for this paper was carried out using the Liverpool JMU and Sussex University Starlink Nodes. JSC wishes to acknowledge a PPARC research award.

\section{References}

Clark, J. S., \& Steele, I. A. 2000, A\&AS, 141, 65, Paper II Dougherty, S. M., \& Taylor, A. R. 1992, Nature, 359, 808 Gehrz, R. D., Hackwell, J. A., \& Jones, T. W. 1974, ApJ, 191, 675

Hamann, F., \& Persson, S. E. 1989, ApJS, 71, 931

Hanson, M. M., Conti, P. S., \& Rieke, G. H. 1996, ApJS, 107, 281

Hanson, M. M., Rieke, G. H., \& Luhman, K. L. 1998, AJ, 116, 1915

Hanuschik, R. W. 1989, Astron. Space Sci., 161, 61
Hony, S., et al. 2000, A\&A, 355, 187

Howells, L., Steele, I. A., Porter, J. M., \& Etherton, J. 2001, A\&A, 369, 99

Koornneef, J. 1983, A\&A, 128, 84

Millar, C. E., \& Marlborough, J. M. 1998, ApJ, 494, 715

Marlborough, J. M., Zijlstra, J.-W., \& Waters, L. B. F. M. 1997, A\&A, 321, 867

Porter, J. M. 1996, MNRAS, 280, L31

Quirrenbach, A., Buscher, D. F., Mozurkewich, D., Hummel, C. A., \& Armstrong, J. T. 1994, A\&A, 283, 13

Scargle, J. D., et al. 1978, ApJ, 224, 527

Stee, P., de Araujo, F. X., Vakili, F., et al. 1995, A\&A, 300, 219

Steele, I. A., Negueruela, I., \& Clark, J. S. 1999, A\&AS, 137, 147, Paper I

Steele, I. A., \& Clark, J. S. 2001, MNRAS, submitted

Storey, P. J., \& Hummer, D. G. 1995, MNRAS, 272, 41 